\title{
Investigating Machine Learning Techniques for Detection of Depression Using Structural MRI Volumetric Features
}

\author{
Kuryati Kipli, Abbas Z. Kouzani, and Isredza Rahmi A. Hamid
}

\begin{abstract}
Structural MRI offers anatomical details and high sensitivity to pathological changes. It can demonstrate certain patterns of brain changes present at a structural level. Research to date has shown that volumetric analysis of brain regions has importance in depression detection. However, such analysis has had very minimal use in depression detection studies at individual level. Optimally combining various brain volumetric features/attributes, and summarizing the data into a distinctive set of variables remain difficult. This study investigates machine learning algorithms that automatically identify relevant data attributes for depression detection. Different machine learning techniques are studied for depression classification based on attributes extracted from structural MRI (sMRI) data. The attributes include volume calculated from whole brain, white matter, grey matter and hippocampus. Attributes subset selection is performed aiming to remove redundant attributes using three filtering methods and one hybrid method, in combination with ranker search algorithms. The highest average classification accuracy, obtained by using a combination of both SVM-EM and IG-Random Tree algorithms, is $85.23 \%$. The classification approach implemented in this study can achieve higher accuracy than most reported studies using sMRI data, specifically for detection of depression.
\end{abstract}

Index Terms-MRI, brain image analysis, image feature selection, machine learning, depression detection.

\section{INTRODUCTION}

Depression is the most common mental disorder worldwide and currently the fourth largest contributor to the burden of disease as reported by the World Health Organization [1]. It is estimated that by 2020, depression will remain a leading cause of disability, second only to cardiovascular disease [1]. Depression is associated with widely varying psychological and physiological features, and this heterogeneity is acknowledged within classification systems [2]. Diagnostic criteria for major depressive disorder (MDD) are currently based on clinical and psychometric assessment. The main procedures for evaluation of patients in the field of MDD are neuropsychological screening tests. Some widely used screening tests for the evaluation of depression include Hamilton Rating Scale for Depression (HRSD), Diagnostic Interview Schedule, and Hospital Anxiety and Depression Scale.

Manuscript received February 5, 2013; revised May 16, 2013. This work was supported in part by Deakin University and Ministry of Higher Education Malaysia (MoHE) through SLAB Ph.D scholarship.

K. Kipli and A. Z. Kouzani are with the School of Engineering, Deakin University, Waurn Ponds, Victoria 3216, Australia (e-mail: kkipli@deakin.edu.au).

I. R. A. Hamid is with the School of Information Technology, Deakin University, Waurn Ponds, Victoria 3216, Australia (email: iraha@deakin.edu.au).
The objective of this study is to provide an automated tool to help in diagnosis of depression by differentiating between healthy and depressed patients in sMRI data. Evaluation of sMRI of the brain is usually achieved through visual ratings performed by medical experts (i.e., radiologists, neuroradiologists). However, conventional evaluation of these scans often relies on manual reorientation, visual reading, and semiquantitative analysis of certain regions of the brain. These steps are difficult, time consuming, subjective, and prone to error. In practice, no clinical expert would diagnose brain diseases only by looking at the abnormality of a single region of the brain. Instead, clinical experts carry out a comprehensive visual inspection of every part of the brain. Therefore, an automated detection system is warranted. Automated tools can be applied to anticipate the diagnosis, and avoid the inter and intra rater variability observed when pathologists give different relative important to each of the grading criteria. The focus of this study is to investigate machine learning techniques, including attribute selection and classification. Attribute selection aims at retaining only the most relevant attributes and thus improve the generalization ability and the performance of the classifier [3].

In this study, we explore various parts of the brain using the sMRI imaging data by extracting volumetric attributes from the regions and assessing the significance of each attribute during classification. The attributes extraction has been done by the database provider [4] whereas the purpose here is to focus on the attribute selection and classification. In this work, we also compare the performance of the attribute selection and classifier algorithms by using the accuracy rate. To the authors knowledge, this is the first study that explores machine learning algorithms for depression classification from volumetric attributes. In summary, the contributions of this paper include: 1) evaluating and determining the most discriminant sMRI volumetric attributes for single-subject classification of depression 2) identifying machine learning algorithms that automatically determine relevant attributes and are optimal for depression detection.

This paper is organized as follows. Section II describes the related works. Section III explains the methods and the algorithms, as well as the selected attributes. Section IV describes the proposed experimental procedure comprising the system flowchart. Section V presents the experimental results and discusses them. The conclusions are given in Section VI.

\section{RELATED WORKS}

In group-level analysis, depression is mainly characterized by volumetric reductions or increase in the hippocampus, amygdala, anterior cingulate cortex, orbitofrontal cortex, 
dorsolateral prefrontal cortex, subgenual prefrontal cortex, putamen, and caudate. Also cerebrospinal fluid have been specifically associated with depression. However, the available individual depression detection studies based on sMRI (Costafreda et al. [5], Nouretdinov et al. [6], Gong et al. [7], Mwangi et al. [8], [9], Bao et al. [10]) have utilized different attributes for the detection. The attributes used are voxel based morphometry, brain shape and voxels value.

Attribute selection processes still have not gained much attention in the available depression detection research. There are only few studies that reported on attribute selection process. The reported studies on the investigation of attribute selection from sMRI depression data implemented ANOVA [92] and $t$-test on VBM [8]. Costafreda et al. [5] implemented the whole-brain analysis of variance filtering to select the areas of maximum group differences between patients and controls. Mwangi et al. [8] implemented an attribute selection $t$-test filter in VBM to identify the voxels that differed most in depressed patients versus healthy controls. They also investigated a wrapper method called Recursive Feature Elimination. In other brain imaging studies, Principal Component Analysis (PCA) was employed by Fu et al. [11] and Marquand et al. [12] for attribute selection. The PCA is appealing because it reduces the dimensionality of the data and therefore reduces the computational cost of analyzing new data. Mwangi et al. [8] used relevance vector regression (RVR) that is a sparse algorithm that employs only a fraction of its basis functions to make predictions. A study by Chyzhyk et al. [13] employed Lattice Independent Component Analysis (LICA) and the kernel transformation hybrid with dendritic computing classifiers. These previous studies did not specifically investigate attribute selection.

At the classification, the SVM classifier was employed by Costafreda et al. [5], Gong et al. [7] and Bao et al. [10]. Besides the SVM classifier, Bao et al. [10] also investigated the K-Nearest Neighbor classifier for predicting treatment remission in MDD. Nouretdinov et al. [6] proposed a general probabilistic classification method to structural and functional MRI to investigate diagnostic and prognostic prediction in depression. The proposed method of classification is known as transductive conformal predictors (TCP). Mwangi et al. [9] used regression analysis based on relevance vector regression which is a sparse Bayesian leaning method to predict brain disease. In another published study, Mwangi et al. [8] investigated both RVM and SVM machine learning for diagnostic purpose.

\section{METHODS}

\section{A. Description of the Data, Definition of ROIs and Attribute Extraction}

The 3-D volumetric attributes were extracted from sMRI data provided by Neuropsychiatric Imaging Research Laboratory at Duke University called MIRIAD [4], a NIMH-supported study that has enrolled older depressed and non-depressed adult participants. A total of 115 brain data were included consisting of 88 healthy controls and 27 depressed images. Forty-four volumetric attributes were used for investigation (i.e whole brain volume, gray matter volume, white matter volume, hippocampus volume and etc). A list of attributes and their definition is available in Appendix I. The extracted ROIs were manually traced by an expert neuroradiologist using the Analyze tool, and volumes measured using MrX tools. More detail on the pre-processing is given in ref. [14].

\section{B. Attribute Selection, Ranker Search and Classifier Algorithms}

Four attribute selection algorithms are used including one rule (OneR), support vector machine (SVM), information gain (IG) and ReliefF. In this study we applied default setting for the algorithms run in WEKA, a non-commercial and open-source data mining system [15].

The OneR algorithm creates one rule for each attribute in the training data, then selects the rule with the smallest error rate as its 'one rule'. To create a rule for an attribute, the most frequent class for each attribute value must be determined. The most frequent class is simply the class that appears most often for that attribute value. Finally, it chooses the attribute that offers rules with minimum error and constructs the final decision tree [16].

The SVM evaluates the worth of an attribute by using an SVM classifier [17]. The SVM is that the weights of the decision function are a function only of a small subset of the training examples, called "support vectors". Those are the examples that are closest to the decision boundary and lie on the margin. The existence of such support vectors is at the origin of the computational properties of the SVM and its competitive classification performance.

The IG evaluates the worth of an attribute by measuring the information gain with respect to the class. The information gain is equal to the total entropy for an attribute if for each of the attribute values a unique classification can be made for the result attribute.

The ReliefF evaluates the worthiness of an attribute by repeatedly sampling an instance and considering the value of the given attribute for the nearest instance of the same and different class [18]. It can operate on both discrete and continuous class data.

The Ranker Search algorithm is an extension of the standard forward selection/best first that allows for either a fixed set (i.e. select no more than $\mathrm{n}$ attributes) or a fixed width (consider only adding an attribute from the top $\mathrm{n}$ ranked attributes to the current subset at each step) approach to be used. Both these options result in a faster search than standard forward selection (they give similar and sometimes better results due to less overfitting) [15], [19].

There are many classification algorithms that can be used for classification. In this paper, we used ten different classifiers: Naïve Bayes, SVM RBF, SVM Sigmoid, J48, Random Forest, Random Tree, VFI, LogitBoost, Simple KMeans Classification Via Clustering (KMeans), and Classification Via Clustering EM (EM).

\section{EXPERIMENTAL PROCEDURES}

The experiments are conducted using WEKA, a non-commercial and open-source data mining system [2], [3]. WEKA contains tools for data pre-processing, classification, regression, clustering, association rules, and visualization. It is also well suited for developing new machine learning schemes. Attributes selection is performed using a cross validation strategy with 10 folds and 1 seed. 
1) Volumetric attributes are selected (see Appendix I) from the sMRI dataset (44 attributes are selected in this work). From the selected attributes we constructed a data matrix.

2) Class values are assigned as nominal/binary $(0,1)$ values; 0 represents healthy subjects and 1 stands for depressed subjects.

3) All the attributes are ranked using four attribute selection methods.

4) The attribute rankings are tabulated from highest to lowest for the four methods.

5) New datasets are created from each attribute ranking result; A total of 17 new matrixes are formed out of the original data matrix as follows: Top 1, Top 2, Top 3, Top 4, Top 5, Top 6, Top 7, Top 8, Top 9, Top 10, Top 15, Top 20, Top 25, Top 30, Top 35, Top 40, and Top 43.

6) The new data subsets are named according to format; Top $<$ Ranking No. $><$ Attribute Selection Name $>$. Each attribute selection has 17 sets, thus, there are 68 new dataset formed from this combination.

7) Ten classifiers are selected for the classification: Naïve Bayes, SVM RBF, SVM Sigmoid, J48, Random Forest, Random Tree, VFI, LogitBoost, Simple KMeans Classification Via Clustering, and Classification Via Clustering EM. The classification experiments were designed as training/test of 70/30. All together we have 68 $\times 10=680$ possible combination. Instances were "arranged to ensure balanced no of class in each sub set". The classification rule set to follow the initial arrangement (with order preserved).

8) The accuracies percentages for the classification were automatically calculated by WEKA. The results are ranked in descending order using the accuracy percentage.

\section{RESUlTS AND DisCUSSIONS}

\section{A. Classification Performances}

Table I shows the accuracy rates (percentage of correctly classified samples) and the average area under the receiver operating characteristic curve (AUC), respectively, for the combination that achieved accuracy greater than or equal to $80 \%$. The results are sorted from the highest to lowest accuracies. It can be seen that the hybrid evaluator SVM and the filter evaluator IG has the highest accuracy.

The best accuracy for the attribute selection-classifier is displayed in Fig. 1. When the presented attribute selection methods were used in combination with an SVM $\mathrm{RBF} /$ Sigmoid, the results were consistent regardless of the attribute selection used. The evaluator OneR in combination with SVM RBF classifier produced slightly higher accuracy of $79.41 \%$ compared to the other methods. These results were comparable or better than those reported in previous works [5], [7], [8], [10] using the SVM. The existing works reported accuracies between $58.70 \%$ to $87.1 \%$ when using the SVM, while our classification showed stable results between $76.47 \%$ to $79.41 \%$. However, the accuracy increased when an EM or Random Tree classifier was applied instead of the SVM. The hybrid evaluator SVM in combination with the EM classifier and the filter evaluator IG in combination with the Random Tree classifier recorded highest accuracy of $85.29 \%$. The good performance of the EM classifier here attributed to the significantly small number of attributes, $N=7$ needed for the classification. Overall, it can be seen that the EM, J48, Random Forest and Random Tree classifiers achieved good performances and have the potential for depression detection classification problem. However, the Naïve Bayes and the VFI performances were very sensitive to the attribute selection used.

Finally, the proposed attribute selection was compared against the previous works [5], [8] which used statistical filter method (ANOVA and $t$-test). The classification accuracy in ref. [5] is $67.6 \%$, which is smaller than the accuracy of the proposed attribute selection. However, the classification accuracy in [8] is $87.1 \%$, which is slightly higher than the accuracy of the proposed attribute selection. The performance difference may also be due to the difference in the attributes used.

TABLE I: AVERAgE ACCURACy (ACC), Number of AtTRIBUTES (N), F-MEASURE AND AREA UNDER THE CURVE (AUC)

\begin{tabular}{lllll}
\hline \hline Evaluator & Classifier & N & Accuracy & AUC \\
\hline SVM & EM & 7 & 85.2941 & 0.6875 \\
IG & RandomTree & 15 & 85.2941 & 0.6875 \\
ReliefF & J48 & 30 & 82.3529 & 0.4856 \\
IG & J48 & 35 & 82.3529 & 0.4856 \\
SVM & Kmeans & 1 & 82.3529 & 0.6683 \\
SVM & Kmeans & 7 & 82.3529 & 0.6683 \\
All & NaiveBayes & 44 & 82.3529 & 0.5962 \\
SVM & RandomForest & 8 & 82.3529 & 0.7163 \\
IG & RandomForest & 6 & 82.3529 & 0.7188 \\
IG & RandomForest & 15 & 82.3529 & 0.6875 \\
ReliefF & RandomTree & 3 & 82.3529 & 0.6250 \\
ReliefF & RandomTree & 5 & 82.3529 & 0.6250 \\
\hline \hline
\end{tabular}

\section{B. Evaluation of Attributes/Features}

The attributes of the final set are different for each classification pair and each attribute selection method. Table II shows the most frequent selected attributes at certain achieved accuracy. The table tabulates the attributes with its corresponding frequency and ranked in descending order. From Table II, it can be seen that the Top 2 attributes contributed to accuracy $\geq 75-85$ are the same and the Top 4 attributes contributed to accuracy $\geq 75$ and 80 are the same. For the accuracy score of 75 or above, the Top 1 (ltotgm) in the ranking doubled the frequency for attributes on ranked number 11 (rhippoc) and 12 (rtotgm).

Specifically, when the accuracy $\geq 80$, the Top 1 and 2 have significantly higher frequencies compared to the rest. The frequency reduced significantly from Top 2 to Top 3 attributes. Interestingly, for higher accuracy (85\%), we could perceive that only 18 attributes contributing and the frequency for the Top 4 attributes is actually doubled the remaining. Specifically for accuracy $\geq 85 \%$, left-brain dominated the contribution with total frequency of 14 frequencies. Fig. 2 illustrates the most important brain attributes for the accuracy $\geq 85 \%$. This result is in accordance to reported works in depression at group-level statistical analysis. For example, previous studies have shown a morphometric reduction of the hippocampus (bilateral hippocampus, left and right hippocampus, and hippocampus 
grey matter) in patients with depression compared to healthy important attribute was ltotgm. controls [20]. It can be seen that on overall, the most

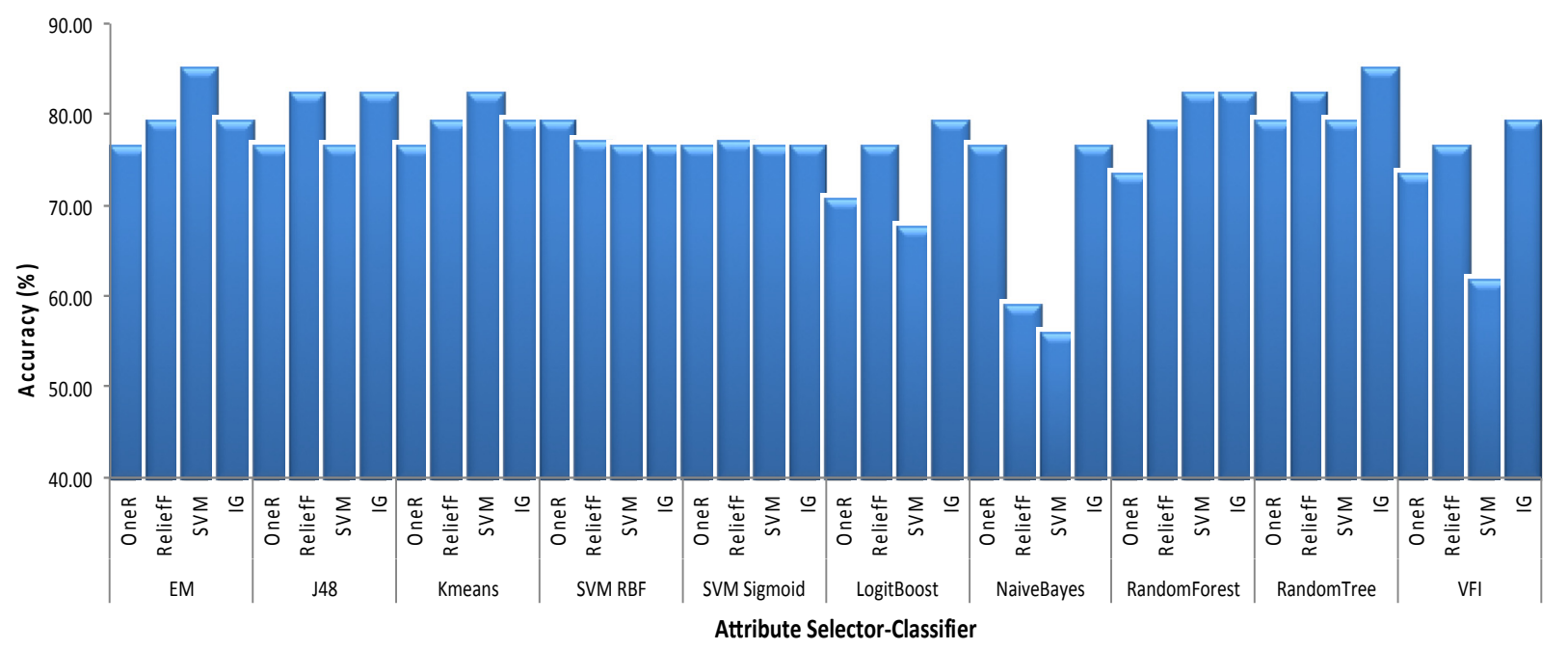

Fig. 1. Best classification accuracy of attribute selection-classifier combination.

TABLE II: The Most FreQuent SELECTED Attributes AND THE

\begin{tabular}{|c|c|c|c|c|c|c|}
\hline \multicolumn{3}{|c|}{$\mathrm{ACC} \geq 75$} & \multicolumn{2}{|c|}{$\mathrm{ACC} \geq 80$} & \multicolumn{2}{|c|}{$A C C \geq 85$} \\
\hline No & Attributes & $f$ & Attributes & $f$ & Attributes & $f$ \\
\hline 1 & ltotgm & 204 & ltotgm & 10 & ltotgm & 2 \\
\hline 2 & lhemis & 194 & lhemis & 9 & lhemis & 2 \\
\hline 3 & lnonlgm & 182 & lnonlgm & 7 & ltotcsf & 2 \\
\hline 4 & ltotcsf & 145 & ltotesf & 7 & nvesf & 2 \\
\hline 5 & wholebr & 144 & nvcsf & 7 & lnonlgm & 1 \\
\hline 6 & cerebrm & 131 & wholebr & 6 & wholebr & 1 \\
\hline 7 & tothippoc & 128 & $\operatorname{lnvesf}$ & 5 & $\operatorname{lnvesf}$ & 1 \\
\hline 8 & $\operatorname{lnvesf}$ & 127 & lvent & 5 & lvent & 1 \\
\hline 9 & nvesf & 116 & rhippoc & 5 & rhippoc & 1 \\
\hline 10 & lvent & 113 & ltotwm & 5 & ltotwm & 1 \\
\hline 11 & rhippoc & 109 & lgmles & 4 & lgmles & 1 \\
\hline 12 & rtotgm & 96 & totgm & 4 & totgm & 1 \\
\hline 13 & ltotwm & 91 & nonlgm & 4 & nonlgm & 1 \\
\hline 14 & lgmles & 91 & lnonlwm & 4 & lnonlwm & 1 \\
\hline 15 & totgm & 84 & totvent & 4 & totvent & 1 \\
\hline 16 & totcsf & 82 & cerebrm & 3 & ltotles & 1 \\
\hline 17 & nonlgm & 80 & tothippoc & 3 & rgmles & 1 \\
\hline 18 & rgmltc & 78 & ltotles & 3 & lwmles & 1 \\
\hline 19 & rnonlgm & 76 & rgmles & 3 & cerebrm & 0 \\
\hline 20 & ltotles & 75 & lwmles & 3 & tothippoc & 0 \\
\hline
\end{tabular}

*ACC: accuracy

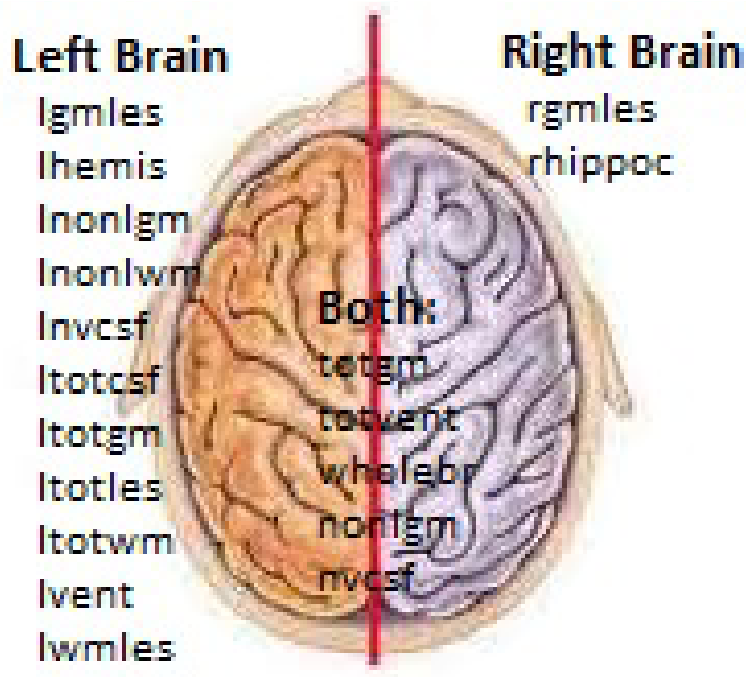

Fig. 2. The most important brain attributes $(A C C \geq 85 \%)$.

\section{CONCLUSION}

In this study, several machine learning techniques for attribute selection and classification were examined for depression detection using the brain volumetric attributes. The potential of attributes extracted from the brain sMRI volumetric calculation was explored and the diagnostic value of each attribute was investigated. The performance results highlight the potential of depression detection from sMRI volumetric attributes. The SVM evaluator in combination with the EM classifier and the IG evaluator in combination with the Random Tree classifier have achieved the highest accuracy. However, the small sample sizes limits the ability to draw firm conclusions. Thus, further studies with larger datasets are necessary to generalize the results and improve the performance of the whole detection system.

\section{APPENDIX}

APPENDIX I: VOLUMETRIC ATTRIBUTES DESCRIPTIONS

\begin{tabular}{|c|c|c|}
\hline No & Attributes & Description \\
\hline 1 & nonlgm & $\begin{array}{l}\text { Non-lesion gray matter (GM) volume in whole } \\
\text { brain }\end{array}$ \\
\hline 2 & gmles & $\begin{array}{l}\text { Subcortical gray matter lesion (GML) volume in } \\
\text { cerebrum }\end{array}$ \\
\hline 3 & totgm & Total GM volume \\
\hline 4 & nvesf & Non-ventricular CSF volume in the whole brain \\
\hline 5 & totvent & Total Lateral ventricle volume \\
\hline 6 & totcsf & Total CSSF volume \\
\hline 7 & nonlwm & $\begin{array}{l}\text { Non-lesion white matter (WM) volume in whole } \\
\text { brain }\end{array}$ \\
\hline 8 & wmles & WM lesion volume in the cerebrum \\
\hline 9 & totwm & total WM volume \\
\hline 10 & totles & total lesion volume \\
\hline 11 & wholebr & $\begin{array}{l}\text { whole brain volume } \\
\text { Non-lesion GM volume in left cerebral }\end{array}$ \\
\hline 12 & lnonlgm & $\begin{array}{l}\text { hemisphere } \\
\text { Subcortical GML volume in the left cerebral }\end{array}$ \\
\hline 13 & lgmles & hemisphere \\
\hline 14 & ltotgm & $\begin{array}{l}\text { left hemisphere total GM volume } \\
\text { Non-ventricular CSF volume in left cerebral }\end{array}$ \\
\hline 15 & $\operatorname{lnvesf}$ & hemisphere \\
\hline 16 & lvent & hemisphere \\
\hline 17 & ltotcsf & left hemisphere total CSF volume \\
\hline 18 & lnonlwm & $\begin{array}{l}\text { Non-lesion WM volume in left cerebral } \\
\text { hemisphere }\end{array}$ \\
\hline 19 & lwmles & WML volume in the left cerebral hemisphere \\
\hline 20 & ltotwm & left hemisphere total WM volume \\
\hline 21 & ltotles & left hemisphere total lesion volume \\
\hline
\end{tabular}




\begin{tabular}{|c|c|c|}
\hline 22 & lhemis & left hemisphere volume \\
\hline & rnonlgm & $\begin{array}{l}\text { Non-lesion GM volume in right cerebral } \\
\text { hemisphere }\end{array}$ \\
\hline & & Subcortical GML volume in the right cerebral \\
\hline & rgmles & hemisphere \\
\hline 25 & rtotgm & $\begin{array}{l}\text { right hemisphere total GM volume } \\
\text { Non-ventricular CSF volume in the right cerebral }\end{array}$ \\
\hline 6 & rnvesf & $\begin{array}{l}\text { hemisphere } \\
\text { Lateral ventricle volume in the right cerebral }\end{array}$ \\
\hline 27 & rvent & hemisphere \\
\hline 28 & rtotcsf & $\begin{array}{l}\text { right hemisphere total CSF volume } \\
\text { Non-lesion WM volume in right cerebral }\end{array}$ \\
\hline 29 & rnonlwm & hemisphere \\
\hline 30 & rwmles & WML volume in the right cerebral hemisphere \\
\hline 31 & rtotwm & right hemisphere total WM volume \\
\hline 32 & rtotles & right hemisphere total lesion volume \\
\hline 33 & rhemis & right hemisphere volume \\
\hline 34 & cerebrm & cerebral volume \\
\hline 35 & lgmtc & Left caudate GM volume \\
\hline 36 & lgmltc & Left caudate lesion volume \\
\hline 37 & rgmtc & Right caudate GM volume \\
\hline 38 & rgmltc & Right caudate lesion volume \\
\hline 39 & lputamn & Left putamen volume \\
\hline 40 & rputamn & Right putamen volume \\
\hline 41 & lhippoc & Left hippocampus volume \\
\hline 42 & rhippoc & Right hippocampus volume \\
\hline 43 & totputamn & total putamen volume \\
\hline 4 & tothippoc & total hippocampus volume \\
\hline
\end{tabular}

\section{ACKNOWLEDGMENT}

We thank the Duke University NIRL for making the MIRIAD MRI data available."

\section{REFERENCES}

[1] J. Sayers, "The world health report 2001-mental health: new understanding, new hope," Bull World Health Organ., vol. 79, pp. 1085, 2001.

[2] A. M. Gruenberg, R. D. Goldstein, and H. A. Pincus, "Classification of depression: Research and diagnostic criteria: DSM-IV and ICD-10," Biology of Depression, Wiley-VCH Verlag GmbH, 2008, pp. 1-12.

[3] E. Zacharaki, V. Kanas, and C. Davatzikos, "Investigating machine learning techniques for MRI-based classification of brain neoplasms," International Journal of Computer Assisted Radiology and Surgery, vol. 6 , pp. 821-828, 2011.

[4] NIRL Imaging Database. [Online]. Available: http://nirlarc.duhs.duke.edu/nirle/

[5] S. G. Costafreda, C. Chu, J. Ashburner, and C. H. Y. Fu, "Prognostic and diagnostic potential of the structural neuroanatomy of depression," PLoS ONE, vol. 4, pp. e6353, 2009.

[6] I. Nouretdinov, S. G. Costafreda, A. Gammerman, A. Chervonenkis, V. Vovk, V. Vapnik, and C. H. Y. Fu, "Machine learning classification with confidence: Application of transductive conformal predictors to MRI-based diagnostic and prognostic markers in depression,' NeuroImage, vol. 56, pp. 809-813, 2011.

[7] Q. Gong, Q. Wu, C. Scarpazza, S. Lui, Z. Jia, A. Marquand, X. Huang, P. McGuire, and A. Mechelli, "Prognostic prediction of therapeutic response in depression using high-field MR imaging," NeuroImage, vol. 55, pp. 1497-1503, 2011.

[8] B. Mwangi, K. P. Ebmeier, K. Matthews, and J. Douglas Steele, "Multi-centre diagnostic classification of individual structural neuroimaging scans from patients with major depressive disorder," Brain, vol. 135, pp. 1508-1521, May 1, 2012.

[9] B. Mwangi, K. Matthews, and J. D. Steele, "Prediction of illness severity in patients with major depression using structural MR brain scans," Journal of Magnetic Resonance Imaging, vol. 35, pp. 64-71, 2012.

[10] F. Bao, S. Ghosh, J. Giard, R. Parsey, and A. Klein, "Brain shape analysis for predicting treatment remission in major depressive disorder," presented at the 41 st Annual Meeting for the Society for Neuroscience, 2011.

[11] C. H. Y. Fu, J. Mourao-Miranda, S. G. Costafreda, A. Khanna, A. F. Marquand, S. C. R. Williams, and M. J. Brammer, "Pattern classification of sad facial processing: toward the development of neurobiological markers in depression," Biological Psychiatry, vol. 63, pp. 656-662, 2008.

[12] A. F. Marquand, J. Mourão-Miranda, M. J. Brammer, A. J. Cleare, and C. H. Y. Fu, "Neuroanatomy of verbal working memory as a diagnostic biomarker for depression," NeuroReport, vol. 19, pp. 1507-1511, 2008.
[13] D. Chyzhyk, M. Graña, A. Savio, and J. Maiora, "Hybrid dendritic computing with kernel-LICA applied to Alzheimer's disease detection in MRI," Neurocomputing, vol. 75, pp. 72-77, 2012.

[14] D. C. Steffens, C. E. Byrum, D. R. McQuoid, D. L. Greenberg, M. E. Payne, T. F. Blitchington, J. R. MacFall, and K. R. R. Krishnan, "Hippocampal volume in geriatric depression," Biological Psychiatry, vol. 48, pp. 301-309, 2000

[15] E. Frank, M. Hall, L. Trigg, G. Holmes, and I. H. Witten, "Data mining in bioinformatics using Weka," Bioinformatics, vol. 20, pp. 2479-2481, October 12, 2004.

[16] S. Ali and K. A. Smith, "On learning algorithm selection for classification," Applied Soft Computing, vol. 6, pp. 119-138, 2006.

[17] I. Guyon, J. Weston, S. Barnhill, and V. Vapnik, "Gene selection for cancer classification using support vector machines," Mach. Learn. vol. 46, pp. 389-422, 2002.

[18] M. Šikonja and I. Kononenko, "Theoretical and empirical analysis of reliefF and RreliefF," Mach. Learn., vol. 53, pp. 23-69, 2003.

[19] R. Ruiz, J. Riquelme, and J. Aguilar-Ruiz, "Heuristic search over a ranking for feature selection computational intelligence and bioinspired systems," J. Cabestany, A. Prieto, and F. Sandoval, Eds., Springer Berlin / Heidelberg, vol. 3512, pp. 498-503, 2005.

[20] K. Kipli, A. Kouzani, and L. Williams, "Towards automated detection of depression from brain structural magnetic resonance images," Neuroradiology, pp. 1-18, 2013.

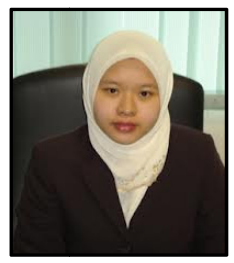

Kuryati Kipli received the B.Eng. degree in electronic and computer engineering from the Universiti Malaysia Sarawak (UNIMAS), Malaysia, in 2004, and the M.Sc. degree in electronic and computer engineering from the University of Birmingham, U.K in 2007. She is currently working toward the Ph.D degree at the School of Engineering, Deakin University, Waurn Ponds, Australia.

From 2004 to 2006, she was a process integration engineer at the $1^{\text {st }}$ Silicon (M'sia) Sdn Bhd, Samajaya Industrial, Sarawak, Malaysia. From 2006 - 2007, she was a tutor at the Faculty of Engineering, Universiti Malaysia Sarawak. From 2008 to date, she worked in the Department of Electronics, Faculty of Engineering, UNIMAS. She is now on study-leave from the Department of Electronics, Faculty of Engineering, UNIMAS. Since 2008, she has authored and coauthored more than 35 publications (including journal publications, conference publications and book chapter). Her current research interests include pattern recognition, image processing and biomedical image analysis.

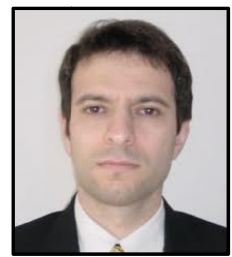

Abbas Z. Kouzani received his M.Eng. degree in electrical and electronics engineering from the University of Adelaide, Australia, 1995, and Ph.D. degree in electrical and electronics engineering from Flinders University, Australia, 1999. He was a lecturer with the School of Engineering, Deakin University, Australia, and then a senior lecturer with the School of Electrical Engineering and Computer Science, University of Newcastle, Australia. Currently, he is an Associate Professor with the School of Engineering, Deakin University, Australia.

Associate Professor Kouzani has been involved in over $\$ 1.9$ million research grants including Australian national competitive, industry, and university grants. He has published over 190 refereed papers. He served as the associate head of school (research) in the School of Engineering, Deakin University for several years. Also, he was the leader of the Electronics, Mechatronics, and Robotics stream with the same School. He is an OzReader with the Australian Research Council, and reviews for a number of international journals and conferences. He has also carried out applied research and consultancy for several Australian and international companies. His research interests include various aspects of BioMEMS in particular medical/biological microdevices, lab-on-a-chip systems, bioinstrumentation, biosensors and implants, embedded wireless microdevices, etc. He is the leader of Deakin University's BioMEMS Research Group.

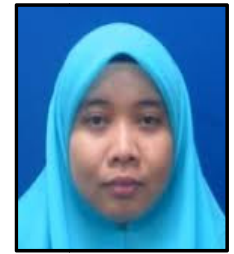

Isredza Rahmi A. Hamid is currently attached to Deakin University, Australia, as a post graduate student Faculty of Science, Engineering and Built Environment. She is a faculty member of Information Security Department, Faculty Science Computer and Information Technology, University Tun Hussein Onn Malaysia. She received her M.Sc. from MARA University of Technology (Malaysia), and Bachelor

Degree in Information Technology from Northern University Malaysia (Malaysia). She has published several papers in reviewed conferences as well as technical reports. Her research interest includes Network Security (Phishing Detection and classification). 